\title{
On the Properties of Acyclic Bilateral Phase Type Distributions*
}

\author{
András Horváth \\ Dipartimento di Informatica, Università di Torino \\ horvath@di.unito.it
}

\author{
Miklós Telek \\ Department of Telecommunications, Technical \\ University of Budapest \\ telek@hit.bme.hu
}

\begin{abstract}
This paper aims to collect a set of properties of bilateral phase type $(\mathrm{BPH})$ distributions that helps their practical use. Similar to the relation of the phase type $(\mathrm{PH})$ and the acyclic phase type (APH) distributions, it turns out that restricting the underlying Markov chain to be acyclic results in a more tractable class of distributions, the acyclic bilateral phase type $(\mathrm{ABPH})$ distributions. Here we present a canonical representation, moment bounds and moment matching methods for $\mathrm{ABPH}$ distributions.
\end{abstract}

\section{Keywords}

phase type distributions, bilateral phase type distributions, moment bounds, moment fitting.

\section{INTRODUCTION}

Because of their simplicity and tractability Markovian models are widely used in applied stochastic modelling. For the first sight, it is a limitation of Markov models that the inter-event times of continuous time Markov chains (CTMC) are exponentially distributed, but a set of exponentially distributed events controlled by a background CTMC can be used to model non-exponential durations. The class of distributions composed this way is referred to as phase type $(\mathrm{PH})$ distributions (see, e.g. $[12,11])$. Phase type distributions find application in a wide range of fields and they are also effectively applied to approximate general (non-PH) non-negative distributions (a non-complete list of references: $[2,4,10,7,8,6,9])$.

To extend the applicability of Markov modulated models in distribution fitting beyond non-negative distributions, Ahn and Ramaswami recently introduced the class of bilateral phase type $(\mathrm{BPH})$ distributions [1]. This class of

* This work is partially supported by the Italian-Hungarian bilateral R\&D programme, by OTKA grant n. T-34972 and by MIUR through PRIN project Famous.

Permission to make digital or hard copies of all or part of this work for personal or classroom use is granted without fee provided that copies are not made or distributed for profit or commercial advantage and that copies bear this notice and the full citation on the first page. To copy otherwise, to republish, to post on servers or to redistribute to lists, requires prior specific permission and/or a fee.

SMCTools '07, October 26, 2007, Nantes, France

Copyright 2007 ICST 978-963-9799-00-4. continuous distributions (assuming no probability mass at zero) can have support on $(0, \infty),(-\infty, 0)$ or $(-\infty, \infty)$.

Together with the definition of BPH distributions the authors posed several research problems in [1]. In this paper we consider some of these problems and extend the classification with the class of acyclic bilateral phase type (ABPH) distributions. Throughout this paper we consider the case with support on $(-\infty, \infty)$ with no probability mass at zero. The specialisation of the results to the other cases follows the same pattern as the similar treatment of $\mathrm{PH}$ distributions.

The rest of the paper is organised as follows. Section 2 introduces the class of bilateral phase type distributions. Section 3 presents the class of acyclic bilateral phase type distributions and some of its properties. Symbolic moment matching expressions and moment bounds are provided in Section 4 and 5, respectively. Section 6 presents a moment matching method, which generate $\mathrm{BPH}$ distributions based on their first 3 moments, and Section 7 demonstrates the behaviour of this method.

\section{BILATERAL PHASE TYPE DISTRIBU- TIONS}

Following [1] we define the class of BPH distribution with the help of Markov reward models.

Let $\{Z(t), t \geq 0\}$ be a continuous time Markov chain (CTMC) on state space $S=\{1,2, \ldots, N+1\}$ with generator $\mathbf{Q}=\left\{q_{i j}\right\}$ and initial probability vector $\gamma$. The state space is composed by $N$ transient state $\{1,2, \ldots, N\}$ and an absorbing one $\{N+1\}$.

We assign a real valued constant, referred to as reward rate, to the states of $S$ and a real valued reward function, $B(t)$, to $Z(t)$ such that $B(t)$ describes the reward accumulated by $Z(t)$ in the interval $(0, t) . B(0)=0$ and during the sojourn in state $i \in S$ the amount of accumulated reward increases at rate $r_{i}$, i.e., $d B(t) / d t=r_{i}$, when $Z(t)=i$. If $r_{i}$ is negative $B(t)$ decreases during the sojourn in $i$. The amount of reward accumulated during the interval $(0, t)$ is

$$
B(t)=\int_{0}^{t} r_{Z(\tau)} d \tau
$$

Let $R$ be the diagonal matrix composed of the reward rates of the transient states, $R=\operatorname{diag}\left\langle r_{i}\right\rangle$.

The time to absorption of the CTMC, $\tau=\inf \{t \mid Z(t)=$ $N+1\}$, is said to be phase type distributed with initial probability vector $\alpha$, composed by the elements of $\gamma$ associated with the transient states, and generator $A$, composed by the 
elements of $Q$ associated with the transient states:

$$
\gamma=[\alpha, \bullet] ; \quad Q=\left[\begin{array}{cc}
A & \bullet \\
0 & 0
\end{array}\right] .
$$

Definition 1. [1] The reward accumulated till absorption, $B(\tau)$, is bilateral phase type (BPH) distributed with initial probability vector $\alpha$, transient generator $A$ and reward matrix R.

[1] presents several properties of BPH distributions:

1. The class of BPH distributions inherits several useful properties of $\mathrm{PH}$ distributions like denseness, closure (for finite sum, mixture, linear combination), tractability.

2. The $(\alpha, A, R)$ representation is not unique. E.g., if $C$ is a non-singular diagonal matrix with positive entries, then $(\alpha, C A, C R)$ defines the same $\mathrm{BPH}$ distribution. Thanks to this property all rates can be changed to 1 or -1 without changing the distribution.

3. One can eliminate the states with zero reward rates.

4. One of the most interesting properties of BPH distributions is that they can be composed as the mixture of a positive and a negative $\mathrm{PH}$ distribution.

A consequence of this composition, not mentioned in [1], is that the $\mathrm{BPH}$ class also inherits closure property for minimum and maximum operation.

\subsection{Moments of BPH distributions}

Based on property 4 we introduce probability $p$, which is the probability that the $\mathrm{BPH}$ distribution is positive, and 2 (positive) $\mathrm{PH}$ distributions, $\mathrm{PH}^{+}$and $\mathrm{PH}^{-}$. The $\mathrm{BPH}$ takes the value of $\mathrm{PH}^{+}$with probability $p$ and $(-1) * \mathrm{PH}^{-}$with probability $1-p$. Let $\mu_{i}, \mu_{i}^{+}$, and $\mu_{i}^{-}$be the $i$ th moment the $\mathrm{BPH}, \mathrm{PH}^{+}$and $\mathrm{PH}^{-}$distributions, respectively. With this notation the moments of the BPH distribution satisfy

$$
\mu_{i}=p \mu_{i}^{+}+(-1)^{i}(1-p) \mu_{i}^{-} .
$$

\subsection{Continuity behaviour at zero}

The potential discontinuity of the probability density function (pdf) of BPH distributions is mentioned in [1]. Practical applications of $\mathrm{BPH}$ distributions might require that their pdf functions exhibit a given continuity behaviour at zero. In particular we pay special attention to those $\mathrm{ABPH}$ distributions whose pdf has equal left and right limit at zero. These distributions will be referred to as ABPH-C.

\section{ACYCLIC BILATERAL PHASE TYPE DISTRIBUTIONS}

To extend the applicability and tractability of BPH distributions we introduce the subclass with acyclic underlying Markov chain.

Definition 2. The BPH distribution, whose underlying Markov chain does not contain cycle (i.e., $A$ is an upper triangular matrix) is referred to as acyclic bilateral phase type $(A B P H)$ distribution.
Indeed $\mathrm{ABPH}$ distributions inherit the properties of the APH distributions, such as denseness, closure (for finite sum, mixture, linear combination, $\min / \max$ operation), tractability, real eigenvalues and we provide further consequences below.

$\operatorname{ABPH}\left(n^{+}, n^{-}\right)$denotes an acyclic bilateral phase type distribution for which the number of phases with positive (negative) rate is $n^{+}\left(n^{-}\right)$, where $n^{+} \geq 0\left(n^{-} \geq 0\right)$.

Theorem 3. Any ABPH $\left(n^{+}, n^{-}\right)$distribution can be transformed into the canonical form depicted in Figure 1.

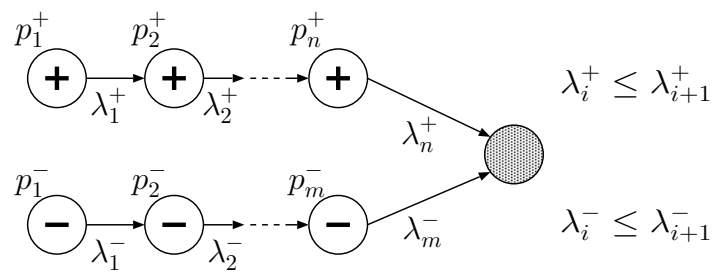

Figure 1: Canonical form of an $\operatorname{ABPH}\left(n^{+}, n^{-}\right)$distribution with $n^{+}=n$ and $n^{-}=m$

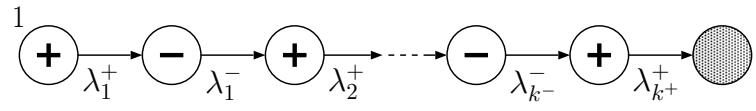

Figure 2: A path of an ABPH distribution

Proof. Consider a path with $k^{-} \leq n^{-}$negative and $k^{+} \leq n^{+}$positive phases leading from an initial state to the absorbing state as depicted in Figure 2. The path can be reorganised in such way, as illustrated in Figure 3, that at first the phases with positive rate are passed through. This transformation obviously does not modify the probability density of the fluid accumulated along the path. The same probability density function can be realized by the structure depicted in Figure 4. The initial probabilities of the negative phases have to be set as

$$
\begin{aligned}
& p_{i}^{-}=\operatorname{Pr}\{\text { fluid accumulated along the path of Figure } 3 \\
& \text { is negative and fluid level crosses level zero } \\
& \text { during the stay in the } i \text { th negative phase. }\}
\end{aligned}
$$

The initial probabilities of the positive phases can be determined by interchanging the role of the negative and positive phases.

The decomposition into negative and positive parts described above can be performed for all possible paths of the $\operatorname{ABPH}\left(n^{+}, n^{-}\right)$distribution. By applying the results presented in [5], the probabilistic mixture of the negative (positive) parts of all paths can be realized by a single sequence of $n^{+}$positive $\left(n^{-}\right.$negative) phases; this sequence is in canonical form. The combination of the sequence with negative

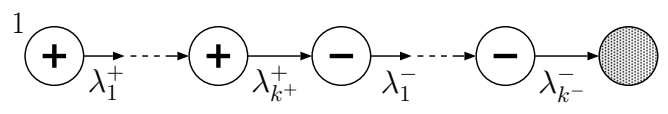

Figure 3: Reorganised path of an ABPH distribution 


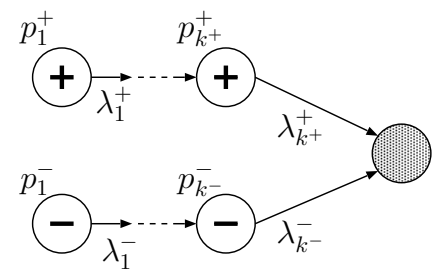

Figure 4: Path of an ABPH distribution decomposed into negative and positive parts

phases and the sequence with positive phases results in the structure depicted in Figure 1 with associated constraints which is a canonical form for $\operatorname{ABPH}\left(n^{+}, n^{-}\right)$distributions. $\square$

\section{SYMBOLIC EXPRESSIONS FOR 3 MO- MENT MATCHING}

\subsection{Moments matching $\mathrm{ABPH}(1,1)$}

Assuming there is no probability mass at zero the first 3 moments define the parameters of an $\operatorname{ABPH}(1,1)$ based on (2) which takes the form

$$
\mu_{k}=k ! p\left(\lambda^{+}\right)^{-k}+k !(1-p)\left(\lambda^{-}\right)^{-k}, \quad k=1,2,3 .
$$

(4) simplify to a second order equation, whose relevant solution is

$$
\begin{aligned}
p= & \frac{-27 \mu_{1}^{2} \mu_{2}^{2}+18 \mu_{2}^{3}+\mu_{3}\left(\mu_{3}-\sqrt{c}\right)}{2 c} \\
& +\frac{-12 \mu_{1}^{3}\left(-2 \mu_{3}+\sqrt{c}\right)+9 \mu_{1} \mu_{2}\left(-2 \mu_{3}+\sqrt{c}\right)}{2 c}, \\
\lambda^{-} & =\frac{-3 \mu_{1} \mu_{2}+\mu_{3}+\sqrt{c}}{3 \mu_{2}^{2}-2 \mu_{1} \mu_{3}}, \lambda^{+}=\frac{3 \mu_{1} \mu_{2}-\mu_{3}+\sqrt{c}}{3 \mu_{2}^{2}-2 \mu_{1} \mu_{3}},
\end{aligned}
$$

where $c=-27 \mu_{1}^{2} \mu_{2}^{2}+18 \mu_{2}^{3}+24 \mu_{1}^{3} \mu_{3}-18 \mu_{1} \mu_{2} \mu_{3}+\mu_{3}^{2}$. The $\mu_{1}, \mu_{2}, \mu_{3}$ moments are feasible $\operatorname{ABPH}(1,1)$ moments (exists an $\operatorname{ABPH}(1,1)$, whose first 3 moments are $\left.\mu_{1}, \mu_{2}, \mu_{3}\right)$, if the solution is feasible, i.e., $0 \leq p \leq 1, \lambda^{+}>0, \lambda^{-}>0$.

\subsection{Moments matching with ABPH-C $(1,1)$ distributions}

The density of an $\operatorname{ABPH}(1,1)$ is

$$
f(t)= \begin{cases}(1-p) \lambda^{-} e^{\lambda^{-} t} & \text { if } t<0 \\ p \lambda^{+} e^{-\lambda^{+} t} & \text { if } t>0\end{cases}
$$

where the $\lambda^{+}>0$ and $\lambda^{-}>0$, are the parameters of the positive and the negative parts and $p(0<p<1)$ is the mixing probability.

Applying (4) for $k=1,2$ and considering that the pdf has to have equal left and right limits, i.e. $p \lambda^{+}=(1-p) \lambda^{-}$, results in 3 equations for the three unknowns. There are two solutions of this set of equation and the relevant one is:

$$
\begin{gathered}
p=\frac{3 \mu_{1}^{2}-2 \mu_{2}-\mu_{1} \sqrt{2 \mu_{2}-3 \mu_{1}^{2}}}{6 \mu_{1}^{2}-4 \mu_{2}}, \\
\lambda^{+}=\frac{-\mu_{1}+\sqrt{2 \mu_{2}-3 \mu_{1}^{2}}}{\mu_{2}-2 \mu_{1}^{2}}, \lambda^{-}=\frac{\mu_{1}+\sqrt{2 \mu_{2}-3 \mu_{1}^{2}}}{\mu_{2}-2 \mu_{1}^{2}},
\end{gathered}
$$

The $\mu_{1}, \mu_{2}, \mu_{3}$ moments are feasible ABPH-C $(1,1)$ moments, if the solution is feasible, i.e., $0 \leq p \leq 1, \lambda^{+}>0, \lambda^{-}>0$.

\section{MOMENT BOUNDS FOR 3 MOMENTS}

The previous section presented results where the first 3 moments, or the moments and the continuity behaviour uniquely determine the ABPH distribution. In this section we extend the 3 moments based analysis for higher order BHP distributions, but in this case the 3 moments do not uniquely define the distribution. First we provide results on the permissable range of the tree moments and than in the next section a fitting procedure to create $\mathrm{BPH}$ distributions with a given first 3 moments.

\subsection{Moment bounds for ABPH distributions}

Let $n_{2}=\frac{\mu_{1}^{2}}{\mu_{2}}$ and $n_{3}=\frac{\mu_{1} \mu_{2}}{\mu_{3}}$ denote the second and third normalized moments of a distribution with ordinary moments $\mu_{1}, \mu_{2}, \mu_{3}$. The following theorem is from [3].

Theorem 4. The second and third normalized moments of the APH(n) class are subjects to the following constraints

$$
\frac{n+1}{n} \leq n_{2}<\infty,
$$

$n_{3}$ lower bound:

$$
\begin{cases}l_{n} \leq n_{3} & \text { if } \frac{n+1}{n} \leq n_{2} \leq \frac{n+4}{n+1}, \\ \frac{n+1}{n} n_{2}<n_{3} & \text { if } \frac{n+4}{n+1}<n_{2},\end{cases}
$$

$n_{3}$ upper bound:

$$
\begin{cases}n_{3} \leq u_{n} & \text { if } \frac{n+1}{n} \leq n_{2} \leq \frac{n}{n-1}, \\ n_{3}<\infty & \text { if } \frac{n}{n-1}<n_{2},\end{cases}
$$

where $l_{n}$ and $u_{n}$ are defined as follows:

$$
\begin{gathered}
l_{n}=\frac{\left(3+a_{n}\right)(n-1)+2 a_{n}}{(n-1)\left(1+a_{n} p_{n}\right)} \\
-\frac{2 a_{n}(n+1)}{2(n-1)+a_{n} p_{n}\left(n a_{n}+2 n-2\right)}, \\
u_{n}=\frac{1}{n^{2} n_{2}}\left(2(n-2)\left(n n_{2}-n-1\right) \sqrt{1+\frac{n\left(n_{2}-2\right)}{n-1}}\right. \\
\left.+(n+2)\left(3 n n_{2}-2 n-2\right)\right)
\end{gathered}
$$

with

$$
\begin{gathered}
p_{n}=\frac{(n+1)\left(n_{2}-2\right)}{3 n_{2}(n-1)}\left(\frac{-2 \sqrt{n+1}}{\sqrt{4(n+1)-3 n n_{2}}}-1\right), \\
a_{n}=\frac{n_{2}-2}{p_{n}\left(1-n_{2}\right)+\sqrt{p_{n}^{2}+\frac{p_{n} n\left(n_{2}-2\right)}{n-1}}} .
\end{gathered}
$$

Let $\mathrm{ABPH}^{+}\left(n, n^{-}\right)$denote an $\mathrm{ABPH}\left(n^{+}, n^{-}\right)$distribution with $n^{+}=n, n^{-} \geq 1$ (at least one negative phase), and $\mu_{1}>0$ (positive first moment).

Theorem 5. The second and third normalised moments of an $\mathrm{ABPH}^{+}\left(n, n^{-}\right)$distribution are subject to the following constraints:

$$
\frac{n+1}{n} \leq n_{2}<\infty
$$




$$
\begin{array}{ll}
-\infty<n_{3} \leq u_{n} & \text { if } \frac{n+1}{n} \leq n_{2} \leq \frac{n}{n-1}, \\
-\infty<n_{3}<\infty & \text { if } \frac{n}{n-1}<n_{2},
\end{array}
$$

where $u_{n}$ is given in (9) in Theorem 4. Note that

- the bounds for the second normalised moment are identical for both the $A P H(n)$ class and the $A B P H^{+}\left(n, n^{-}\right)$ class,

- the upper bound for the third normalised moment is identical for both the $A P H(n)$ and the $A B P H^{+}\left(n, n^{-}\right)$,

- the lower bound for the third normalised moment for the $A B P H^{+}\left(n, n^{-}\right)$class is $-\infty$,

- the bounds does not depend on the number of negative phases, i.e., a single negative phase is sufficient to reach the bounds.

Proof. Thanks to Theorem 3 we can assume that the $\mathrm{ABPH}^{+}\left(n, n^{-}\right)$distribution is given by a mixture of an $\operatorname{ABPH}\left(n^{+}, 0\right)$ with $n^{+}=n$ and an $\operatorname{ABPH}\left(0, n^{-}\right)$distribution. Let $\mu_{1}^{+}, \mu_{2}^{+}, \mu_{3}^{+}, n_{2}^{+}, n_{3}^{+}\left(-\mu_{1}^{-}, \mu_{2}^{-},-\mu_{3}^{-}, n_{2}^{-}, n_{3}^{-}\right)$denote the moments and the normalised moments of the $\operatorname{ABPH}(n, 0)\left(\operatorname{ABPH}\left(0, n^{-}\right)\right)$distribution. Then the moments of the $\mathrm{ABPH}^{+}\left(n, n^{-}\right)$distribution satisfy (2). By introducing $a=\mu_{1}^{+} / \mu_{1}^{-}$the normalised moments of the $\mathrm{ABPH}^{+}\left(n, n^{-}\right)$distribution are

$$
\begin{aligned}
& n_{2}=\frac{n_{2}^{-}(1-p)+a^{2} n_{2}^{+} p}{(-(1-p)+a p)^{2}}, \\
& n_{3}=\frac{-n_{2}^{-} n_{3}^{-}(1-p)+a^{3} n_{2}^{+} n_{3}^{+} p}{(-(1-p)+a p)\left(n_{2}^{-}(1-p)+a^{2} n_{2}^{+} p\right)} .
\end{aligned}
$$

Since the first moment of $\operatorname{ABPH}^{+}\left(n, n^{-}\right)$is positive we must have

$$
p \mu_{1}^{+}-(1-p) \mu_{1}^{-}>0 \text { and since } p>\frac{1}{1+a} .
$$

The second normalised moment given in (12) is monotone decreasing in the interval $p \in(1 /(1+a), 1]$ and its limits are

$$
\lim _{p \rightarrow\left(\frac{1}{1+a}\right)^{+}} n_{2}=\infty, \quad \lim _{p \rightarrow 1} n_{2}=n_{2}^{+} .
$$

According to $(6) n_{2}^{+}$is bounded by $(n+1) / n \leq n_{2}^{+}<\infty$ and since (15) implies (10). The only value of $p$ that satisfies (12) and (14) is

$$
\begin{aligned}
& p=\frac{1}{2(1+a)^{2} n_{2}}\left(2(1+a) n_{2}-n_{2}^{-}+a^{2} n_{2}^{+}+\right. \\
& \left.\sqrt{n_{2}^{-}\left(4 a(1+a) n_{2}+n_{2}^{-}\right)+2 a^{2}\left(2(1+a) n_{2}-n_{2}^{-}\right) n_{2}^{+}+a^{4} n_{2}^{+2}}\right) .
\end{aligned}
$$

Substituting (16) into (13), it can be shown that $n_{3}$ is a monotone increasing function of $a$ in the interval $a \in(0, \infty)$ with limits

$$
\lim _{a \rightarrow 0^{+}} n_{3}=-\infty, \quad \lim _{a \rightarrow \infty}=\frac{n_{2} n_{3}^{+}}{n_{2}^{+}}
$$

which implies the lower bound in (11) for the third normalised moments of an $\mathrm{ABPH}^{+}\left(n, n^{-}\right)$distribution. For what concerns the upper bound, based on Theorem 4 and (15) we have $(n+1) / n \leq n_{2}^{+} \leq n_{2}$. Theorem 4 implies also that in the range $n_{2+} \in\left[(n+1) / n, n_{2}\right]$ the quantity $n_{2} n_{3+} / n_{2+}$ is maximal if $n_{2}=n_{2+}$ and $n_{3+}$ is the maximal third normalised moment realizable by an $\operatorname{APH}(n)$ distribution. This implies the upper bound in (11) for the third normalised moment of an $\mathrm{ABPH}^{+}\left(n, n^{-}\right)$distribution which is identical to that of an $\operatorname{APH}(n)$ distribution.

The numerical values of the bounds of the $\mathrm{APH}(\mathrm{n})$ and the $\mathrm{ABPH}^{+}\left(n, n^{-}\right)$class are illustrated in Figure 5 and 6 . The darkest shaded area contains those normalised moments pairs that can be realized with two phases. Points in the second darkest shaded area can be realized with three phases. The dashed dotted line corresponds to $n_{3}=n_{2}$ which is the lower limit of non-negative random variables.

The structure of these bounds are presented in Figure 7 and 8 . These figures indicate the $n_{3}=2 n_{2}-1$ line as well because it plays a special role. The second and third normalised moments of the Erlang(n) $(n \geq 1)$ distributions lie on this line.

Let $\mathrm{ABPH}^{-}\left(n^{+}, n\right)$ denote an $\mathrm{ABPH}\left(n^{+}, n^{-}\right)$distribution with $n^{-}=n, n^{+} \geq 1$ (at least one positive phase) and $\mu_{1}<0$ (negative first moment).

Theorem 6. The second and third normalised moments of an $\mathrm{ABPH}^{-}\left(n^{+}, n\right)$ distribution are subject to the following constraints:

$$
\begin{gathered}
\frac{n+1}{n} \leq n_{2}<\infty, \\
-\infty<n_{3} \leq u_{n} \quad \text { if } \frac{n+1}{n} \leq n_{2} \leq \frac{n}{n-1}, \\
-\infty<n_{3}<\infty \quad \text { if } \frac{n}{n-1}<n_{2},
\end{gathered}
$$

where $u_{n}$ is given in (9) in Theorem 4.

Proof. The theorem is the trivial counterpart of Theorem 5 .

The normalised moments cannot be used in the case when the mean is zero. The following theorem gives the bounds for the second and third moments for this case.

Theorem 7. The bounds for the second and the third moments of a $\mathrm{ABPH}\left(n^{+}, n^{-}\right)$distribution with zero mean are

$$
0<\mu_{2}<\infty \text { and }-\infty<\mu_{3}<\infty \text {. }
$$

Proof. We prove the theorem only for the most restricted case. Consider the $\operatorname{ABPH}(1,1)$ structure depicted in Figure 9. From $\mu_{1}=0$ we have $p / \lambda^{+}-(1-p) / \lambda^{-}=0$, from which $p^{+}=\lambda^{+} /\left(\lambda^{+}+\lambda^{-}\right)$. The second moment then can be written as

$$
\mu_{2}=\frac{2}{\lambda^{-} \cdot \lambda^{+}}=\frac{2 a}{\left(\lambda^{-}\right)^{2}}
$$

where $a=\lambda^{-} / \lambda^{+}$. The limits for the second moment are

$$
\lim _{\lambda^{-} \rightarrow \infty} \mu_{2}=0, \quad \lim _{\lambda^{-} \rightarrow 0} \mu_{2}=\infty .
$$

From (21) we have

$$
\lambda^{-}=\sqrt{\frac{2 a}{\mu_{2}}}
$$




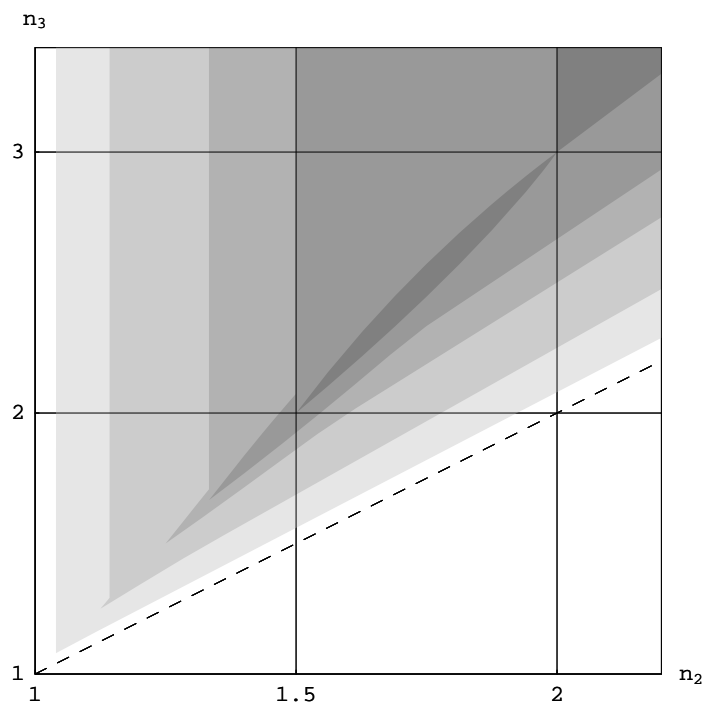

Figure 5: Bounds of normalised moments of the $\mathbf{A P H}(n)$ class $(n=2,3,4,8,25)$

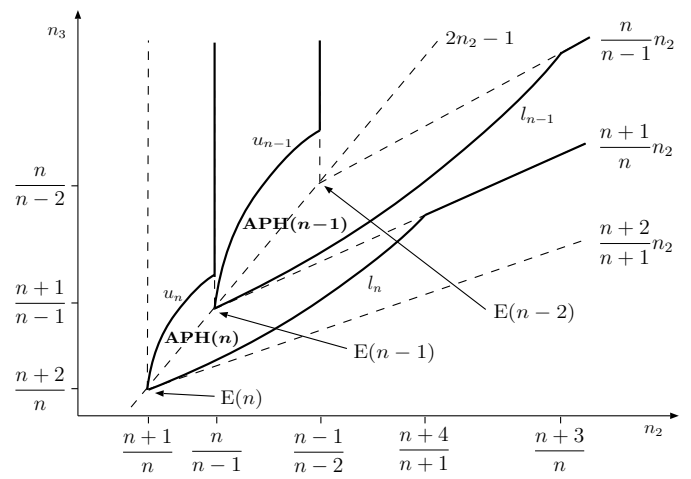

Figure 7: Structure of bounds of normalised moments of the $\operatorname{APH}(n)$ class

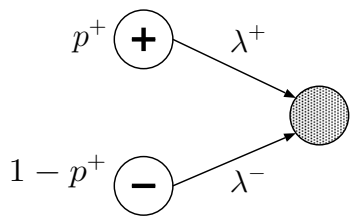

Figure 9: $\operatorname{ABPH}(1,1)$ distribution

with which the third moment is

$$
\mu_{3}=\frac{3(a-1) \mu_{2}^{3 / 2}}{\sqrt{2 a}} .
$$

The limits for the third moment are

$$
\lim _{a \rightarrow 0} \mu_{3}=-\infty, \quad \lim _{a \rightarrow \infty} \mu_{3}=\infty .
$$

Since for any $\operatorname{ABPH}\left(n^{+}, n^{-}\right)$with $\mu_{2}>0$, Theorem 7

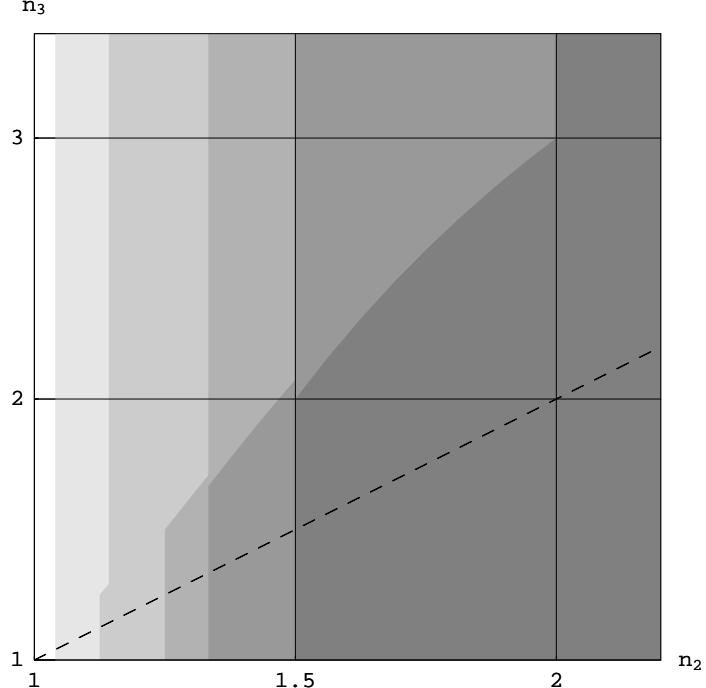

Figure 6: Bounds of normalised moments of the $\mathbf{A B P H}^{+}\left(n^{+}, n^{-}\right)$class $(n=2,3,4,8,25)$

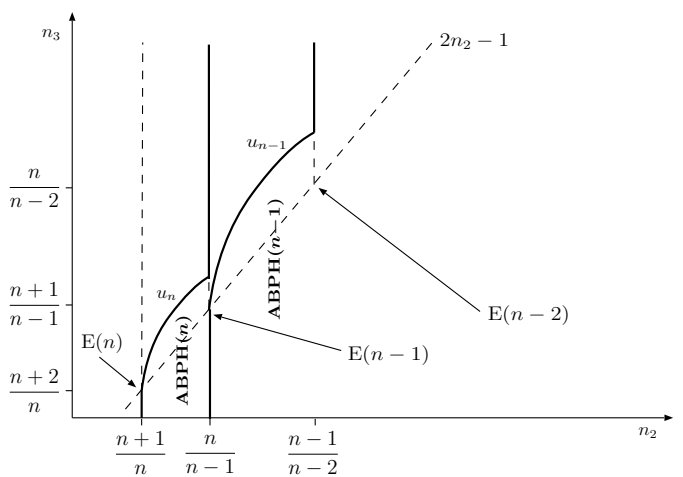

Figure 8: Structure of bounds of normalised moments of the $\mathrm{ABPH}^{+}\left(n^{+}, n^{-}\right)$class

implies that in case of zero mean, the combination of a single negative and a single positive phase gives complete flexibility with respect to the possible range of the second and third normalised moments. Based on (24), given second and third moments, $\mu_{2}$ and $\mu_{3}$, can be realized by

$$
a= \begin{cases}\frac{9 \mu_{2}^{3}+\mu_{3}^{2}-\mu_{3} \sqrt{18 \mu_{2}^{2}+\mu_{3}^{2}}}{9 \mu_{2}^{3}} & \text { if } \mu_{3}<0, \\ \frac{9 \mu_{2}^{3}+\mu_{3}^{2}+\mu_{3} \sqrt{18 \mu_{2}^{2}+\mu_{3}^{2}}}{9 \mu_{2}^{3}} & \text { if } 0 \leq \mu_{3} .\end{cases}
$$

Then $\lambda^{-}$is given by (23), $\lambda^{+}=\lambda^{-} / a$ and, based on the zero mean assumption, $p=1 /(1+a)$.

\subsection{Moment bounds for ABPH-C distribu- tions}

In this section we deal with $\mathrm{ABPH}$ distributions whose pdf has equal left and right limits at zero. A simple sufficient but not necessary condition for having this property is that 


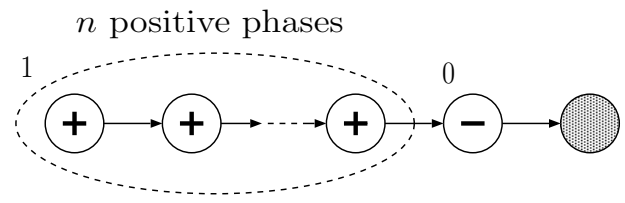

Figure 10: $\mathrm{ABPH}(\mathrm{n}, 1)$ distribution with equal limits of the pdf at zero

every basic path contains both positive and negative phases.

As shown by Theorem 5, a single negative phase is enough to have whole flexibility for the first three moments in case of the $\mathrm{ABPH}^{+}\left(n^{+}, n^{-}\right)$class. The following theorem defines a region of 2 nd and 3rd normalised moments that can be realized with a special $\mathrm{ABPH}-\mathrm{C}^{+}(n, 1)$ structure.

Theorem 8. Any point of the following second and third normalised moment range can be obtained by an $A B P H^{+}(n, 1)$ distribution, depicted in Figure 10, composed of $n$ positive phases and a single negative phase with zero initial probability:

$$
\frac{n+1}{n} \leq n_{2}<\infty,
$$

$$
\begin{array}{ll}
l_{n}<n_{3}<u_{n}, & \text { if } \quad \frac{n+1}{n}<n_{2}<\frac{n}{n-1}, \\
\hat{l}_{n}<n_{3}<\infty, & \text { if } \frac{n}{n-1}<n_{2},
\end{array}
$$

where

$$
\begin{gathered}
\hat{l}_{n}=\frac{3}{1-a_{1}} \\
-\frac{\left(\left(1-a_{1}\right) n_{2}-2\right)^{2}}{n_{2} a_{1}\left(1-a_{1}\right)}\left(2-\frac{a_{1}^{2}}{\left(1-a_{1}\right)\left(\left(1-a_{1}\right) n_{2}-2\right)}\right), \\
a_{1}=\frac{n_{2}-1+\sqrt{\left(n_{2}-1\right) \frac{n+1}{n}-\frac{1}{n}}}{n_{2}-\frac{n+1}{n}},
\end{gathered}
$$

and $l_{n}$ and $u_{n}$ are given in (8) and (9), respectively.

Proof. The moments of the given $\mathrm{ABPH}^{+}(n, 1)$ structure are

$$
\begin{gathered}
\mu_{1}=\mu_{1}^{+}-\mu_{1}^{-}, \mu_{2}=\mu_{2}^{+}+\mu_{2}^{-}-2 \mu_{1}^{+} \mu_{1}^{-}, \\
\mu_{2}=\mu_{3}^{+}-\mu_{3}^{-}-3 \mu_{2}^{+} \mu_{1}^{-}+3 \mu_{1}+\mu_{2}^{-},
\end{gathered}
$$

where $\mu_{1}^{+}>\mu_{1}^{-}$and $\mu_{k}^{-}=k !\left(\mu_{1}^{-}\right)^{k}$. Introducing $a=\mu_{1}^{+} / \mu_{1}^{-}$ and the normalised moments we obtain

$$
\begin{gathered}
n_{2}=\frac{2(1-a)+a^{2} n_{2}^{+}}{(a-1)^{2}}, \\
n_{3}=\frac{6(a-1)-3 a^{2} n_{2}^{+}+a^{3} n_{2}^{+} n_{3}^{+}}{(a-1)\left(2(1-a)+a^{2} n_{2}^{+}\right)} .
\end{gathered}
$$

From $\mu_{1}^{+}>\mu_{1}^{-}$we have $a>1$.

In (29), $n_{2}$ is a monotone decreasing function of $a$ for $a>1$, such that $\lim _{a \rightarrow 1}+n_{2}=\infty$ and $\lim _{a \rightarrow \infty} n_{2}=n_{2}^{+}$, which, together with Theorem 4, means that

$$
\frac{n+1}{n} \leq n_{2}^{+} \leq n_{2}<\infty .
$$

Solving (29) for $n_{2}^{+}$we have

$$
n_{2}^{+}=\frac{(1-a)\left((1-a) n_{2}-2\right)}{a^{2}},
$$

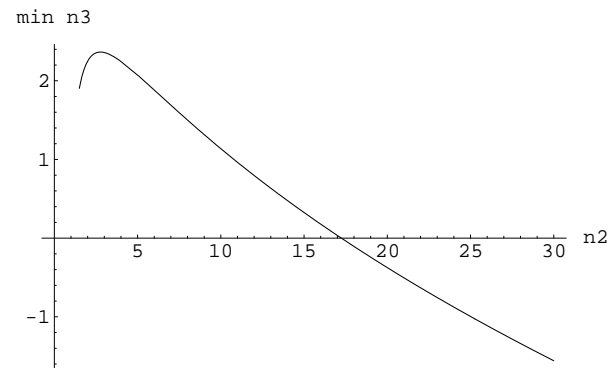

Figure 11: The lower bound of $n_{3}$ when $n_{2}>n /(n-1)$ and $n=3$

from which, for a given $n_{2}, n_{2}^{+}$can take values in $\left(0, n_{2}\right)$, where the lower limit is obtained as $a \rightarrow 1^{+}$and the upper as $a \rightarrow \infty$. Since $n_{2}^{+}$is lower bounded according to (31) (Theorem 4) the valid range of $n_{2}^{+}$is $\left(\frac{n+1}{n}, n_{2}\right)$, and the associated valid range of $a$ is

$$
a \in\left(a_{1}, \infty\right) \text {, where } a_{1}=\frac{n_{2}-1+\sqrt{\left(n_{2}-1\right) \frac{n+1}{n}-\frac{1}{n}}}{n_{2}-\frac{n+1}{n}} .
$$

For small $n_{2}$ values, $n_{2}<\frac{n}{n-1}$, (33) gives a high $a_{1}$ value $\left(a_{1}>n+\sqrt{2 n(n-1)}\right)$, which indicates that the negative part has a negligible role. This way for small $n_{2}$ values we bound $n_{3}$ with the bounds of the positive part, which can be obtained as $a \rightarrow \infty$.

Substituting (32) into (30) gives

$$
n_{3}=\frac{3}{1-a}-\frac{\left((1-a) n_{2}-2\right)^{2}}{n_{2} a(1-a)} \frac{n_{3}^{+}}{n_{2}^{+}} .
$$

In (34), $\frac{3}{1-a}$ is negative and $n_{3}$ is an increasing function of $\frac{n_{3}^{+}}{n_{2}^{+}}$, since $1-a$ is negative. For any $n_{2}>\frac{n}{n-1}$, the $a \rightarrow \infty$ limit results that $n_{2}^{+} \rightarrow n_{2}$ and $n_{3}$ is unbounded from above, because $n_{3}^{+}$is unbounded from above when $n_{2}^{+}>\frac{n}{n-1}$.

The remaining limit $\left(\min n_{3}\right.$ when $\left.n_{2}>\frac{n}{n-1}\right)$ is based on the lower bound of the $\frac{n_{3}^{+}}{n_{2}^{+}}$ratio. According to Theorem 4 $\min \frac{n_{3}^{+}}{n_{2}^{+}} \leq \frac{2 n_{2}^{+}-1}{n_{2}^{+}}$when $\frac{n+1}{n}<n_{2}^{+}$, i.e., $a \in\left(a_{1}, \infty\right)$.

Taking the upper bound of the minimal $\frac{n_{3}^{+}}{n_{2}^{+}}$ratio and substituting it into (32) gives:

$$
\begin{aligned}
& \min n_{3} \leq \frac{3}{1-a}-\frac{\left((1-a) n_{2}-2\right)^{2}}{n_{2} a(1-a)}\left(2-\frac{1}{n_{2}^{+}}\right)= \\
& \frac{3}{1-a}-\frac{\left((1-a) n_{2}-2\right)^{2}}{n_{2} a(1-a)}\left(2-\frac{a^{2}}{(1-a)\left((1-a) n_{2}-2\right)}\right) .
\end{aligned}
$$

(36) is a monotone increasing function of $a$ (when $a>1$ and $n_{2}>1$ ). Substituting the minimal value of $a, a=a_{1}$, gives the missing lower bound of $n_{3}$.

For $n_{2}>n /(n-1)$ and $n=3$ the lower bound of $n_{3}$ is depicter in Figure 11. For small $n_{2}$ values we have a large $a$ (which eliminates the effect of the negative part) and the curve follows the increasing tendency of (7). Instead for large $n_{2}$ values the negative part makes its effect on the 
minimal $n_{3}$ value and at a given point it becomes negative and diverges to $-\infty$ as $n_{2}$ tends to infinity.

\section{MATCHING ALGORITHM THREE MOMENTS}

\subsection{Matching 3 moments with ABPH distri- bution}

Hereinafter we briefly sketch a procedure for the construction of an ABPH distribution with given first three moments, $\mu_{i}, 1 \leq i \leq 3$. The mean is assumed to be positive. If the mean is negative the trivial counterpart of the procedure can be applied.

From Theorem 5 we know that a single negative phase is sufficient. With a single negative phase we have that $n_{2}^{-}=2$ and $n_{3}^{-}=3$. The number of phases on the positive side, $n^{+}$, can be easily determined based on the bounds provided in (10) and (11). It can be seen from (15) and (17) that, in order to construct an $\mathrm{ABPH}$ with given 2nd and 3rd normalised moments, $n_{2}$ and $n_{3}$, we must have

$$
n_{2}^{+}<n_{2} \text { and } \frac{n_{2}^{+} n_{3}}{n_{2}}<n_{3}^{+} .
$$

The lower bound for $n_{2}^{+}$and the upper bound for $n_{3}^{+}$for a given $n^{+}$are provided instead by Theorem 4. Having chosen any pair $\left(n_{2}^{+}, n_{3}^{+}\right)$satisfying the above bounds, it is possible to construct an $\mathrm{ABPH}$ distribution with given first three moments, $\mu_{i}, 1 \leq i \leq 3$. This can be done by solving numerically (12) and (13) for $a$ and $p$. Then $\mu_{1}^{+}$can be obtained from $p \mu_{1}^{+}-(1-p) \mu_{1}^{+} / a=\mu_{1}$ and $\mu_{1}^{-}=\mu_{1}^{+} / a$. As a result, on the negative side we have a single phase with mean $\mu_{1}^{-}$while on the positive side we have $n^{+}$phases with mean $\mu_{1}^{+}$and normalised moments $n_{2}^{+}$and $n_{3}^{+}$. The distribution on the positive side can be constructed by the algorithm presented in [3].

The actual values of $n_{2}^{+}$and $n_{3}^{+}$are chosen based on how closely the resulting distribution fits the mean of the negative and the positive side of the original distribution. I.e., we aim to minimise the relative error

$$
\frac{\left|\mu_{1}^{o+}-\mu_{1}^{+}\right|}{\mu_{1}^{o+}}+\frac{\left|\mu_{1}^{o-}-\mu_{1}^{-}\right|}{\mu_{1}^{o-}}
$$

where $\mu_{1}^{o+}$ and $\mu_{1}^{o-}$ denote the mean of the original distribution on the positive and the negative side, respectively. This relative error is minimised through a numerical search in that region of the $\left(n_{2}^{+}, n_{3}^{+}\right)$pairs which allows for the realization of the given 2 nd and 3rd normalised moments, $n_{2}$ and $n_{3}$.

\subsection{Matching 3 moments with ABPH-C dis- tribution}

An algorithm for matching 3 moments with ABPH-C distributions can be defined based on Theorem 8 . We can proceed as follows. The range for $a$ is given in (33). Having chosen a given value of $a$, based on (29), (30) and $\mu=\mu_{1}^{+}-\mu_{1}^{+} / a$ we can obtain $\mu_{1}^{+}, n_{2}^{+}$and $n_{3}^{+}$; and we also have that $\mu_{1}^{-}=\mu_{1}^{+} / a$. The parameters of the positive phases (Figure 10) then can be obtained based on $\mu_{1}^{+}, n_{2}^{+}$and $n_{3}^{+}$ by applying the algorithm given in [3]. The exit rate of the negative phase instead is simply $1 / \mu_{1}^{-}$. The actual value of $a$ is chosen to minimise the error given in (37).

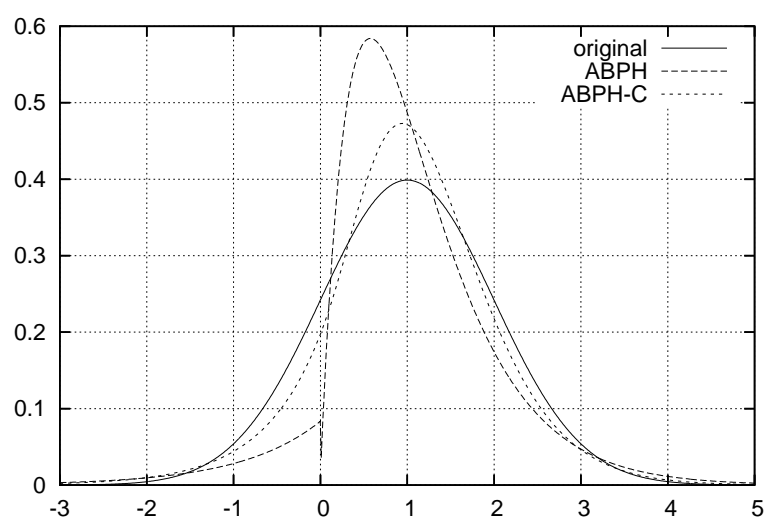

Figure 12: Matching normal distribution with mean and variance equal 1, D1

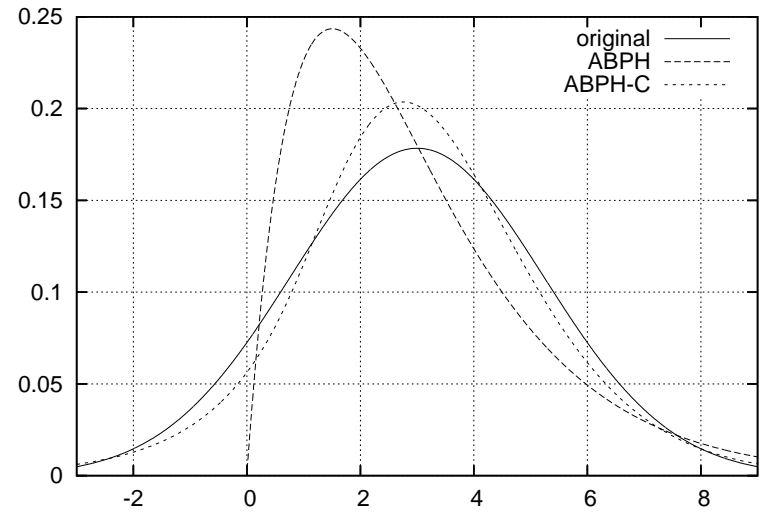

Figure 13: Matching normal distribution with mean equals 3 and variance equals 5, D6

\section{NUMERICAL EXAMPLE}

In this section we consider a few simple examples. The distribution whose normalised moments we match with an $\mathrm{ABPH}$ distribution is the normal distribution. Parameters of the distributions are given in Table 1 which lists: the parameters of the normal distribution (the mean and the variance); the second and the third normalised moments of the normal distribution, $n_{2}$ and $n_{3}$; probability of positive values in the original distribution, $p^{o+}$; the mean of the normal distribution on the negative and on the positive side, $\mu_{1}^{o+}$ and $\mu_{1}^{o-}$.

The distributions are matched by both procedures proposed. Numerical characteristics of the resulting distributions are summarised in Table 2 which lists for both methods: the necessary number of phases on the positive side, $n^{+}$; probability of positive values in the matching distribution, $p^{+}$; the mean of the matching distribution on the negative and the positive side, $\mu_{1}^{+}$and $\mu_{1}^{-}$.

Figures 12 and 13 depict the pdf of two cases, D1 and D6. In Figure 12 it can be seen that we have jump at 0 in the pdf of the matching ABPH distribution. In Figure 13 one can observe instead a case in which the ABPH distribution is not able to fit closely $p^{o+}$. This happens because the region of the $\left(n_{2}^{+}, n_{3}^{+}\right)$pairs from which $n_{2}$ and $n_{3}$ can be realized is very narrow ( $n_{2}^{+}$has to be between 1.5 and 1.5555). In both 


\begin{tabular}{l|ll|ll|lll} 
& $\mu_{1}$ & var. & $n_{2}$ & $n_{3}$ & $p^{o+}$ & $\mu_{1}^{o+}$ & $\mu_{1}^{o-}$ \\
\hline D1 & 1 & 1 & 2 & 2 & 0.8413 & 1.2876 & 0.5251 \\
D2 & 1 & 3 & 4 & 2.5 & 0.7181 & 1.8145 & 1.0752 \\
D3 & 1 & 5 & 6 & 2.667 & 0.6726 & 2.2000 & 1.4657 \\
D4 & 3 & 1 & 1.1111 & 1.2 & 0.9986 & 3.0044 & 0.2831 \\
D5 & 3 & 3 & 1.3333 & 1.5 & 0.9583 & 3.1609 & 0.7034 \\
D6 & 3 & 5 & 1.5555 & 1.7142 & 0.9101 & 3.3985 & 1.0363 \\
D7 & 5 & 1 & 1.04 & 1.0769 & $1-310^{-7}$ & 5 & 0.1865 \\
D8 & 5 & 3 & 1.12 & 1.2143 & 0.9980 & 5.0107 & 0.5045 \\
D9 & 5 & 5 & 1.2 & 1.3333 & 0.9873 & 5.0742 & 0.7777
\end{tabular}

Table 1: Parameters of normal distributions matched by ABPH distributions

\begin{tabular}{l|l|lll|l|lll} 
& \multicolumn{5}{|c}{ ABPH } & \multicolumn{3}{c}{ ABPH-C } \\
\hline & $n^{+}$ & $p^{+}$ & $\mu_{1}^{+}$ & $\mu_{1}^{-}$ & $n^{+}$ & $p^{+}$ & $\mu_{1}^{+}$ & $\mu_{1}^{-}$ \\
\hline D1 & 2 & 0.9231 & 1.1601 & 0.9206 & 5 & 0.8685 & 1.2524 & 0.6656 \\
D2 & 2 & 0.7401 & 1.7324 & 1.0855 & 3 & 0.7625 & 1.6791 & 1.1810 \\
D3 & 2 & 0.6984 & 2.0613 & 1.4576 & 3 & 0.7245 & 1.9953 & 1.6057 \\
D4 & 10 & 0.9973 & 3.0138 & 2.1362 & 18 & 0.9947 & 3.0180 & 0.4100 \\
D5 & 4 & 0.9802 & 3.1037 & 2.1406 & 8 & 0.9697 & 3.1221 & 0.9076 \\
D6 & 2 & 0.9986 & 3.0189 & 10.9965 & 6 & 0.9210 & 3.3352 & 1.2986 \\
D7 & 26 & 0.9999 & 5.0005 & 10.7464 & 38 & $1-210^{-6}$ & 5 & 6.2865 \\
D8 & 9 & 0.9988 & 5.0128 & 5.8747 & 17 & 0.9984 & 5.0119 & 2.4256 \\
D9 & 6 & 0.9914 & 5.0731 & 3.4007 & 12 & 0.9678 & 5.0168 & 0.6839
\end{tabular}

Table 2: Characteristics of the matching ABPH and ABPH-C distributions

case the ABPH-C distribution gives a closer approximation of the pdf of the original distribution, but uses more phases.

Another way of fitting with ABPH distributions is to calculate the first three moments on the negative and on the positive side and perform the fitting of the two sides independently. This would result in higher accuracy $\left(p^{o+}, \mu_{1}^{o+}\right.$ and $\mu_{1}^{o-}$ would be matched perfectly), but requires more phases. For example fitting a normal distribution with mean and variance equal 1 , requires 5 phases if we match the first three moments on both sides while it requires 3 with the procedure proposed in this paper. For what concerns ABPH-C distributions, performing the fitting of the positive and the negative side in separation is not trivial.

\section{REFERENCES}

[1] Soohan Ahn and V. Ramaswami. Bilateral phase type distributions. Stochastic Models, 21:239-259, 2005.

[2] S. Asmussen and O. Nerman. Fitting Phase-type distributions via the EM algorithm. In Proceedings: "Symposium i Advent Statistik", pages 335-346, Copenhagen, 1991.

[3] A. Bobbio, A. Horváth, and M. Telek. Matching three moments with minimal acyclic phase type distributions. Stochastic Models, 21:303-326, 2005.

[4] A. Bobbio and M. Telek. A benchmark for PH estimation algorithms: Results for acyclic-PH. Commun. Statist.-Stochastic Models, 10(3):661-677, 1994.

[5] A. Cumani. On the canonical representation of homogeneous Markov processes modelling failure-time distributions. Microelectronics and Reliability, 22:583-602, 1982.

[6] R. El Abdouni Khayari, R. Sadre, and B. Haverkort. Fitting world-wide web request traces with the EM-algorithm. In Proc. of SPIE, volume 4523, pages 211-220, Denver, USA, 2001.
[7] A. Feldman and W. Whitt. Fitting mixtures of exponentials to long-tail distributions to analyze network performance models. Performance Evaluation, 31:245-279, 1998.

[8] A. Horváth and M. Telek. Approximating heavy tailed behavior with phase type distributions. In $3 r d$ International Conference on Matrix-Analytic Methods in Stochastic models, Leuven, Belgium, 2000.

[9] A. Horváth and M. Telek. Phfit: A general phase-type fitting tool. In Proc. of 12th Performance TOOLS, volume 2324 of Lecture Notes in Computer Science, pages 82-91, Imperial College, London, April 2002.

[10] A. Lang and J. L. Arthur. Parameter approximation for phase-type distributions. In S. R. Chakravarty and A. S. Alfa, editors, Matrix-analytic methods in stochastic models, Lecture notes in pure and applied mathematics, pages 151-206. Marcel Dekker, Inc., 1996.

[11] G. Latouche and V. Ramaswami. Introduction to matrix analytic methods in stochastic modeling. SIAM, 1999.

[12] M. Neuts. Probability distributions of phase type. In Liber Amicorum Prof. Emeritus H. Florin, pages 173-206. University of Louvain, 1975. 